Kragujevac Journal of Mathematics

Volume 41(1) (2017), Pages 143-158.

\title{
ON A CAPUTO FRACTIONAL DIFFERENTIAL INCLUSION WITH INTEGRAL BOUNDARY CONDITION FOR CONVEX-COMPACT AND NONCONVEX-COMPACT VALUED MULTIFUNCTIONS
}

\author{
SH. REZAPOUR ${ }^{1,2, *}$ AND V. HEDAYATI ${ }^{2}$
}

\begin{abstract}
In this paper, we investigate a Caputo fractional differential inclusion with integral boundary condition under different conditions. First, we investigate it for $L^{1}$-Caratheodory convex-compact valued multifunction. Then, we investigate it for nonconvex-compact valued multifunction via some conditions. Also we give two examples to illustrate our results.
\end{abstract}

\section{INTRODUCTION}

As we know, it has been published many papers about the existence of solution for different fractional differential equations (see for example, [8-16] and the references there in). Also, it has been appeared many works on fractional differential inclusions (see for example, $[1-7,18-23,26,29,31-33,36]$ and the references there in). One can find more details about necessary notions in $[25,30,34]$. Let $\eta, \nu, \beta \in(0,1)$ and $\alpha \in(1,2]$ be such that $\Gamma(2-\beta)\left(\eta^{2} \nu-\nu^{2} \eta-\eta^{2}+\nu^{2}+4 \eta-2 \nu-2\right)+2(1-\eta) \neq 0$ and $\alpha-\beta>1$. In this paper, we investigate the existence of solutions for the Caputo fractional differential inclusion

$$
{ }^{c} D^{\alpha} x(t) \in F\left(t, x(t),{ }^{c} D^{\beta} x(t), x^{\prime}(t)\right),
$$

for almost all $t \in[0,1]$, via the integral boundary value conditions

$$
x(0)+x^{\prime}(0)+{ }^{c} D^{\beta} x(0)=\int_{0}^{\eta} x(s) d s
$$

Key words and phrases. Fixed point, fractional differential inclusion, integral boundary value problem.

2010 Mathematics Subject Classification. Primary: 34A60. Secondary: 34B15.

Received: January 28, 2016.

Accepted: September 7, 2016. 
and

$$
x(1)+x^{\prime}(1)+{ }^{c} D^{\beta} x(1)=\int_{0}^{\nu} x(s) d s,
$$

where $F:[0,1] \times \mathbb{R} \times \mathbb{R} \times \mathbb{R} \rightarrow 2^{\mathbb{R}}$ is a compact valued multifunction and ${ }^{c} D^{\alpha}$ is the Caputo differential operator of order $\alpha \in(1,2]$, that is, ${ }^{c} D^{\alpha} x(t)=\frac{1}{\Gamma(2-\alpha)} \int_{0}^{t} \frac{x^{\prime \prime}(s)}{(t-s)^{\alpha-1}} d s$. Let $(X, d)$ be a metric space. It is well known that the Pompeiu-Hausdorff metric (see [17]) $H_{d}: 2^{X} \times 2^{X} \rightarrow[0, \infty)$ is defined by

$$
H_{d}(A, B)=\max \left\{\sup _{a \in A} d(a, B), \sup _{b \in B} d(A, b)\right\}
$$

where $d(A, b)=\inf _{a \in A} d(a, b)$. Then $\left(C B(X), H_{d}\right)$ is a metric space and $\left(C(X), H_{d}\right)$ is a generalized metric space, where $C B(X)$ is the set of closed and bounded subsets of $X$ and $C(X)$ is the set of closed subsets of $X([26])$. Denote the set of compact and convex subsets of $X$ by $P_{c p, c v}(X)$. Let $T: X \rightarrow 2^{X}$ be a multifunction. An element $x \in X$ is called an fixed point of $T$ whenever $x \in T x$ [24]. A multifunction $T: X \rightarrow C(X)$ is called a contraction whenever there exists $\gamma \in(0,1)$ such that $H_{d}(N(x), N(y)) \leq \gamma d(x, y)$ for all $x, y \in X$. In 1970, Covitz and Nadler proved that each closed valued contractive multifunction on a complete metric space has a fixed point [21]. A multifunction $G: J \rightarrow P_{c l}(\mathbb{R})$ is said to be measurable whenever the function $t \mapsto d(y, G(t))$ is measurable for all $y \in \mathbb{R}$, where $J=[0,1]$ [22]. We say that $F: J \times \mathbb{R} \times \mathbb{R} \times \mathbb{R} \rightarrow 2^{\mathbb{R}}$ is a Caratheodory multifunction whenever $t \mapsto F(t, x, y, z)$ is measurable for all $x, y, z \in \mathbb{R}$ and $(x, y, z) \mapsto F(t, x, y, z)$ is upper semi-continuous for almost all $t \in J[7,22,26]$. Also, a Caratheodory multifunction $F: J \times \mathbb{R} \times \mathbb{R} \times \mathbb{R} \rightarrow 2^{\mathbb{R}}$ is called $L^{1}$-Caratheodory whenever for each $\rho>0$ there exists $\phi_{\rho} \in L^{1}\left(J, \mathbb{R}^{+}\right)$such that

$$
\|F(t, x, y, z)\|=\sup \{|v|: v \in F(t, x, y, z)\} \leq \phi_{\rho}(t),
$$

for all $|x|,|y|,|z| \leq \rho$ and for almost all $t \in J[7,22,26]$. By using main idea of $[5,6,31,36]$, we define the set of selections of $F$ by

$$
S_{F, x}:=\left\{v \in A C[0,1](J, \mathbb{R}): v(t) \in F\left(t, x(t),{ }^{c} D^{\beta} x(t), x^{\prime}(t)\right) \text { for almost all } t \in J\right\},
$$

for all $x \in C(J, \mathbb{R})$. Let $E$ be a nonempty closed subset of a Banach space $X$ and $G: E \rightarrow 2^{X}$ a multifunction with nonempty closed values. We say that the multifunction $G$ is lower semi-continuous whenever the set $\{y \in E: G(y) \cap B \neq \emptyset\}$ is open for all open set $B$ in $X$ [24]. It has been proved that each completely continuous multifunction is lower semi-continuous [24]. Denote by $A C[0,1]$ the space of all the absolutely continuous functions defined on $[0,1]$. Let $A C^{2}[0,1]=\left\{w \in C^{1}[0,1]: w^{\prime} \in\right.$ $L[0,1]\}$. Recall that $I^{\alpha} x(t)=\frac{1}{\Gamma(\alpha)} \int_{0}^{t} \frac{x(s)}{(t-s)^{1-\alpha}} d s$ is said to be the Riemann-Liouville fractional integral of order $\alpha$ (for more details see [34,37]). We use the followings in our main results.

Lemma 1.1 ([28]). Let $X$ be a Banach space, $F: J \times X \rightarrow P_{c p, c v}(X)$ an $L^{1}$ Caratheodory multifunction and $\Theta$ a linear continuous mapping from $L^{1}(J, X)$ to 
$C(J, X)$. Then the operator $\Theta_{o} S_{F}: C(J, X) \rightarrow P_{c p, c v}(C(J), X)$ defined by $\left(\Theta o S_{F}\right)(x)$ $=\Theta\left(S_{F, x}\right)$ is a closed graph operator in $C(J, X) \times C(J, X)$.

Lemma 1.2 ([24]). Let $E$ be a Banach space, $C$ a closed convex subset of $E, U$ an open subset of $C$ and $0 \in U$. Suppose that $F: \bar{U} \rightarrow P_{c p, c v}(C)$ is a upper semicontinuous compact map, where $P_{c p, c v}(C)$ denotes the family of nonempty, compact convex subsets of $C$. Then either $F$ has a fixed point in $\bar{U}$ or there exist $u \in \partial U$ and $\lambda \in(0,1)$ such that $u \in \lambda F(u)$.

\section{MAin Results}

Now, we are ready to provide our results about the existence of solutions of the inclusion problem (1.1). Define $x_{v}(t)=I^{\alpha} v(t)-c_{0 v}-c_{1 v} t$, where

$$
\begin{aligned}
c_{0 v}= & -\frac{1}{\Gamma(\alpha)(1-\eta)} \int_{0}^{\eta} \int_{0}^{s}(s-m)^{\alpha-1} v(m) d m d s \\
& +\frac{\left(2-\eta^{2}\right)(\nu-1)}{2 \gamma \Gamma(\alpha)} \int_{0}^{\eta} \int_{0}^{s}(s-m)^{\alpha-1} v(m) d m d s \\
& +\frac{\left(2-\eta^{2}\right)(\eta-1)}{2 \gamma \Gamma(\alpha)} \int_{0}^{1}(1-s)^{\alpha-1} v(s) d s \\
& +\frac{\left(2-\eta^{2}\right)(1-\eta)}{2 \gamma \Gamma(\alpha)} \int_{0}^{\nu} \int_{0}^{s}(s-m)^{\alpha-1} v(m) d m d s \\
& +\frac{\left(2-\eta^{2}\right)(\eta-1)}{2 \gamma \Gamma(\alpha-\beta)} \int_{0}^{1}(1-s)^{\alpha-\beta-1} v(s) d s+\frac{\left(2-\eta^{2}\right)(\eta-1)}{2 \gamma \Gamma(\alpha-1)} \int_{0}^{1}(1-s)^{\alpha-2} v(s) d s
\end{aligned}
$$

and

$$
\begin{aligned}
c_{1 v}= & -\frac{(1-\nu) t}{\gamma \Gamma(\alpha)} \int_{0}^{\eta} \int_{0}^{s}(s-m)^{\alpha-1} v(m) d m d s-\frac{(1-\eta) t}{\gamma \Gamma(\alpha)} \int_{0}^{1}(1-s)^{\alpha-1} v(s) d s \\
& -\frac{(\eta-1) t}{\gamma \Gamma(\alpha)} \int_{0}^{\nu} \int_{0}^{s}(s-m)^{\alpha-1} v(m) d m d s-\frac{(1-\eta) t}{\gamma \Gamma(\alpha-\beta)} \int_{0}^{1}(1-s)^{\alpha-\beta-1} v(s) d s \\
& -\frac{(1-\eta) t}{\gamma \Gamma(\alpha-1)} \int_{0}^{1}(1-s)^{\alpha-2} v(s) d s .
\end{aligned}
$$

It is easy to check that $x_{v} \in A C^{2}[0,1]$ is well-define and $x_{v}^{\prime},{ }^{c} D x_{v}, \int_{0}^{\eta} x_{v}(s) d s$ exist whenever $v \in A C[0,1]$ (see [27]).

Lemma 2.1. Let $v \in A C[0,1], \beta, \eta, \nu \in(0,1)$ and $\alpha \in(1,2]$ with $\alpha-\beta>1$ and

$$
\Gamma(2-\beta)\left(\eta^{2} \nu-\nu^{2} \eta-\eta^{2}+\nu^{2}+4 \eta-2 \nu-2\right)+2(1-\eta) \neq 0 .
$$

Then $x_{v}(t)$ is the unique solution for the problem ${ }^{c} D^{\alpha} x(t)=v(t)$ with the integral boundary value conditions $x(0)+x^{\prime}(0)+{ }^{c} D^{\beta} x(0)=\int_{0}^{\eta} x(s) d s$ and $x(1)+x^{\prime}(1)+$ ${ }^{c} D^{\beta} x(1)=\int_{0}^{\nu} x(s) d s$. 
Proof. It is known that the general solution of the equation ${ }^{c} D^{\alpha} x(t)=v(t)$ is

$$
x(t)=I^{\alpha} v(t)-a_{0}-a_{1} t=\frac{1}{\Gamma(\alpha)} \int_{0}^{t}(t-s)^{\alpha-1} v(s) d s-a_{0}-a_{1} t
$$

where $a_{0}, a_{1}$ are arbitrary constants and $t \in J[25,34]$. Thus,

$$
{ }^{c} D^{\beta} x(t)=I^{\alpha-\beta} v(t)-\frac{t^{1-\beta} a_{1}}{\Gamma(2-\beta)}=\frac{1}{\Gamma(\alpha-\beta)} \int_{0}^{t}(t-s)^{\alpha-\beta-1} v(s) d s-\frac{t^{1-\beta} a_{1}}{\Gamma(2-\beta)}
$$

and $x^{\prime}(t)=I^{\alpha-1} v(t)-a_{1}=\frac{1}{\Gamma(\alpha-1)} \int_{0}^{t}(t-s)^{\alpha-2} v(s) d s-a_{1}$. Hence by using an easy calculation, we get $x(0)+{ }^{c} D^{\beta} x(0)+x^{\prime}(0)=-a_{0}-a_{1}$ and

$$
\begin{aligned}
x(1)+{ }^{c} D^{\beta} x(1)+x^{\prime}(1)= & \frac{1}{\Gamma(\alpha)} \int_{0}^{1}(1-s)^{\alpha-1} v(s) d s \\
& +\frac{1}{\Gamma(\alpha-\beta)} \int_{0}^{1}(1-s)^{\alpha-\beta-1} v(s) d s \\
& \times \frac{1}{\Gamma(\alpha-1)} \int_{0}^{1}(1-s)^{\alpha-2} v(s) d s-\frac{\Gamma(2) a_{1}}{\Gamma(2-\beta)}-2 a_{1}-a_{0} .
\end{aligned}
$$

By using the boundary conditions, we obtain

$$
a_{0}(\eta-1)-a_{1}\left(\frac{\eta^{2}}{2}-1\right)=\frac{1}{\Gamma(\alpha)} \int_{0}^{\eta} \int_{0}^{s}(s-m)^{\alpha-1} v(m) d m d s
$$

and

$$
\begin{aligned}
& a_{0}(\nu-1)+a_{1}\left(\frac{\nu^{2}}{2}-2-\frac{\Gamma(2)}{\Gamma(2-\beta)}\right) \\
= & -\frac{1}{\Gamma(\alpha)} \int_{0}^{1}(1-s)^{\alpha-1} v(s) d s \\
& -\frac{1}{\Gamma(\alpha-\beta)} \int_{0}^{1}(1-s)^{\alpha-\beta-1} v(s) d s-\frac{1}{\Gamma(\alpha-1)} \int_{0}^{1}(1-s)^{\alpha-2} v(s) d s \\
& +\frac{1}{\Gamma(\alpha)} \int_{0}^{\nu} \int_{0}^{s}(s-m)^{\alpha-1} v(m) d m d s .
\end{aligned}
$$

Thus,

$$
\begin{aligned}
a_{0}=c_{0 v}= & -\frac{1}{\Gamma(\alpha)(1-\eta)} \int_{0}^{\eta} \int_{0}^{s}(s-m)^{\alpha-1} v(m) d m d s \\
& +\frac{\left(2-\eta^{2}\right)(\nu-1)}{2 \gamma \Gamma(\alpha)} \int_{0}^{\eta} \int_{0}^{s}(s-m)^{\alpha-1} v(m) d m d s \\
& +\frac{\left(2-\eta^{2}\right)(\eta-1)}{2 \gamma \Gamma(\alpha)} \int_{0}^{1}(1-s)^{\alpha-1} v(s) d s \\
& +\frac{\left(2-\eta^{2}\right)(1-\eta)}{2 \gamma \Gamma(\alpha)} \int_{0}^{\nu} \int_{0}^{s}(s-m)^{\alpha-1} v(m) d m d s
\end{aligned}
$$


ON FRACTIONAL DIFFERENTIAL INCLUSION WITH INTEGRAL BOUNDARY CONDITION47

$$
\begin{aligned}
& +\frac{\left(2-\eta^{2}\right)(\eta-1)}{2 \gamma \Gamma(\alpha-\beta)} \int_{0}^{1}(1-s)^{\alpha-\beta-1} v(s) d s \\
& +\frac{\left(2-\eta^{2}\right)(\eta-1)}{2 \gamma \Gamma(\alpha-1)} \int_{0}^{1}(1-s)^{\alpha-2} v(s) d s
\end{aligned}
$$

and

$$
\begin{aligned}
a_{1}=a_{1 v}= & -\frac{(1-\nu) t}{\gamma \Gamma(\alpha)} \int_{0}^{\eta} \int_{0}^{s}(s-m)^{\alpha-1} v(m) d m d s-\frac{(1-\eta) t}{\gamma \Gamma(\alpha)} \int_{0}^{1}(1-s)^{\alpha-1} v(s) d s \\
& -\frac{(\eta-1) t}{\gamma \Gamma(\alpha)} \int_{0}^{\nu} \int_{0}^{s}(s-m)^{\alpha-1} v(m) d m d s \\
& -\frac{(1-\eta) t}{\gamma \Gamma(\alpha-\beta)} \int_{0}^{1}(1-s)^{\alpha-\beta-1} v(s) d s-\frac{(1-\eta) t}{\gamma \Gamma(\alpha-1)} \int_{0}^{1}(1-s)^{\alpha-2} v(s) d s .
\end{aligned}
$$

Hence,

$$
\begin{aligned}
x(t)= & x_{v} t \\
= & \frac{1}{\Gamma(\alpha)} \int_{0}^{t}(t-s)^{\alpha-1} v(s) d s+\frac{1}{\Gamma(\alpha)(1-\eta)} \int_{0}^{\eta} \int_{0}^{s}(s-m)^{\alpha-1} v(m) d m d s \\
& +\frac{\left(\eta^{2}-2\right)(\nu-1)}{2 \gamma \Gamma(\alpha)} \int_{0}^{\eta} \int_{0}^{s}(s-m)^{\alpha-1} v(m) d m d s \\
& +\frac{\left(\eta^{2}-2\right)(\eta-1)}{2 \gamma \Gamma(\alpha)} \int_{0}^{1}(1-s)^{\alpha-1} v(s) d s \\
& +\frac{\left(\eta^{2}-2\right)(1-\eta)}{2 \gamma \Gamma(\alpha)} \int_{0}^{\nu} \int_{0}^{s}(s-m)^{\alpha-1} v(m) d m d s \\
& +\frac{\left(\eta^{2}-2\right)(\eta-1)}{2 \gamma \Gamma(\alpha-\beta)} \int_{0}^{1}(1-s)^{\alpha-\beta-1} v(s) d s \\
& +\frac{\left(\eta^{2}-2\right)(\eta-1)}{2 \gamma \Gamma(\alpha-1)} \int_{0}^{1}(1-s)^{\alpha-2} v(s) d s \\
& +\frac{(1-\nu) t}{\gamma \Gamma(\alpha)} \int_{0}^{\eta} \int_{0}^{s}(s-m)^{\alpha-1} v(m) d m d s \\
& +\frac{(1-\eta) t}{\gamma \Gamma(\alpha)} \int_{0}^{1}(1-s)^{\alpha-1} v(s) d s+\frac{(\eta-1) t}{\gamma \Gamma(\alpha)} \int_{0}^{\nu} \int_{0}^{s}(s-m)^{\alpha-1} v(m) d m d s \\
& +\frac{(1-\eta) t}{\gamma \Gamma(\alpha-\beta)} \int_{0}^{1}(1-s)^{\alpha-\beta-1} v(s) d s+\frac{(1-\eta) t}{\gamma \Gamma(\alpha-1)} \int_{0}^{1}(1-s)^{\alpha-2} v(s) d s \\
= & I^{\alpha} v(t)-c_{0 v}-c_{1 v} t .
\end{aligned}
$$

Conversely, it is clear that $x_{v}^{\prime}(t)=I^{\alpha-1} v(t)+c_{1 v}$ and $x_{v}^{\prime \prime}(t)=\left(I^{\alpha-1} v(t)\right)^{\prime}={ }^{R} D^{2-\alpha} v(t)$ for almost all $t \in J$. Since $2-\alpha \in(0,1]$, we get

$$
{ }^{c} D^{\alpha} x_{v}(t)=I^{2-\alpha} x_{v}^{\prime \prime}(t)=I^{2-\alpha}\left({ }^{R} D^{2-\alpha} v(t)\right)=v(t) .
$$


Similar to the last part, we obtain

$$
x_{v}(0)+x_{v}^{\prime}(0)+{ }^{c} D^{\beta} x_{v}(0)=-c_{0 v}-c_{1 v}=\int_{0}^{\eta} x(s) d s
$$

and

$$
\begin{aligned}
x_{v}(1)+x_{v}^{\prime}(1)+{ }^{c} D^{\beta} x_{v}(1)= & \frac{1}{\Gamma(\alpha)} \int_{0}^{1}(1-s)^{\alpha-1} v(s) d s \\
& +\frac{1}{\Gamma(\alpha-\beta)} \int_{0}^{1}(1-s)^{\alpha-\beta-1} v(s) d s \\
& \times \frac{1}{\Gamma(\alpha-1)} \int_{0}^{1}(1-s)^{\alpha-2} v(s) d s-\frac{\Gamma(2) a_{1}}{\Gamma(2-\beta)}-2 c_{1 v}-c_{0 v} \\
= & \int_{0}^{\nu} x(s) d s .
\end{aligned}
$$

This completes the proof.

An element $x \in A C^{2}([0,1], \mathbb{R})$ is called a solution of the problem (1.1) whenever it satisfies the integral boundary conditions and there exists a function $v \in S_{F, x}$ such that

$$
x(t)=I^{\alpha} v(t)-c_{0 v}-c_{1 v} t,
$$

for all $t \in J$. Let $X=\left\{x: x, x^{\prime},{ }^{c} D^{\beta} x \in C(J, \mathbb{R})\right.$ for all $\left.\beta \in(0,1)\right\}$ endowed with the norm $\|x\|=\sup _{t \in J}|x(t)|+\sup _{t \in J}\left|{ }^{c} D^{\beta} x(t)\right|+\sup _{t \in J}\left|x^{\prime}(t)\right|$. Then, $(\mathcal{X},\|\|$.$) is a Banach$ space [35]. For investigation of the problem (1.1) we provide two different methods.

Theorem 2.1. Suppose that $F: J \times \mathbb{R} \times \mathbb{R} \times \mathbb{R} \rightarrow P_{c p, c v}(\mathbb{R})$ is a $L^{1}$-Caratheodory multifunction and there exist a bounded continuous non-decreasing map $\psi:[0, \infty) \rightarrow$ $(0, \infty)$ and a continuous function $p: J \rightarrow(0, \infty)$ such that

$$
\left\|F\left(t, x(t),{ }^{c} D^{\beta} x(t), x^{\prime}(t)\right)\right\|=\sup \left\{|v|: v \in F\left(t, x(t),{ }^{c} D^{\beta} x(t), x^{\prime}(t)\right)\right\} \leq p(t) \psi(\|x\|),
$$

for all $t \in J$ and $x \in X$. Then the inclusion problem (1.1) has at least one solution.

Proof. Define the operator $N: X \rightarrow 2^{x}$ by

$N(x)=\left\{h \in \mathcal{X}:\right.$ there exists $v \in S_{F, x}$ such that $\left.h(t)=I^{\alpha} v(t)-c_{0 v}-c_{1 v} t, t \in J\right\}$.

We show that the operator $N$ has a fixed point. First, we show that $N$ maps bounded sets of $\mathcal{X}$ into bounded sets. Suppose that $r>0$ and $B_{r}=\{x \in X:\|x\| \leq r\}$. Let $x \in B_{r}$ and $h \in N(x)$. Choose $v \in S_{F, x}$ such that $h(t)=I^{\alpha} v(t)-c_{0 v}-c_{1 v} t$ for almost all $t \in J$. Thus,

$$
\begin{aligned}
|h(t)| \leq & \frac{1}{\Gamma(\alpha)} \int_{0}^{t}(t-s)^{\alpha-1}|v(s)| d s+\frac{1}{\Gamma(\alpha)(1-\eta)} \int_{0}^{\eta} \int_{0}^{s}(s-m)^{\alpha-1}|v(m)| d m d s \\
& +\left|\frac{\left(\eta^{2}-2\right)(\nu-1)}{2 \gamma \Gamma(\alpha)}\right| \int_{0}^{\eta} \int_{0}^{s}(s-m)^{\alpha-1}|v(m)| d m d s
\end{aligned}
$$


ON FRACTIONAL DIFFERENTIAL INCLUSION WITH INTEGRAL BOUNDARY CONDITION49

$$
\begin{aligned}
& +\left|\frac{\left(\eta^{2}-2\right)(\eta-1)}{2 \gamma \Gamma(\alpha)}\right| \int_{0}^{1}(1-s)^{\alpha-1}|v(s)| d s \\
& +\left|\frac{\left(\eta^{2}-2\right)(1-\eta)}{2 \gamma \Gamma(\alpha)}\right| \int_{0}^{\nu} \int_{0}^{s}(s-m)^{\alpha-1}|v(m)| d m d s \\
& +\left|\frac{\left(\eta^{2}-2\right)(\eta-1)}{2 \gamma \Gamma(\alpha-\beta)}\right| \int_{0}^{1}(1-s)^{\alpha-\beta-1}|v(s)| d s \\
& +\left|\frac{\left(\eta^{2}-2\right)(\eta-1)}{2 \gamma \Gamma(\alpha-1)}\right| \int_{0}^{1}(1-s)^{\alpha-2}|v(s)| d s \\
& +\left|\frac{(1-\nu) t}{\gamma \Gamma(\alpha)}\right| \int_{0}^{\eta} \int_{0}^{s}(s-m)^{\alpha-1}|v(m)| d m d s \\
& +\left|\frac{(1-\eta) t}{\gamma \Gamma(\alpha)}\right| \int_{0}^{1}(1-s)^{\alpha-1}|v(s)| d s+\left|\frac{(\eta-1) t}{\gamma \Gamma(\alpha)}\right| \int_{0}^{\nu} \int_{0}^{s}(s-m)^{\alpha-1}|v(m)| d m d s \\
& +\left|\frac{(1-\eta) t}{\gamma \Gamma(\alpha-\beta)}\right| \int_{0}^{1}(1-s)^{\alpha-\beta-1}|v(s)| d s+\left|\frac{(1-\eta) t}{\gamma \Gamma(\alpha-1)}\right| \int_{0}^{1}(1-s)^{\alpha-2}|v(s)| d s
\end{aligned}
$$

$\leq \Lambda_{1}\|p\|_{\infty} \psi(\|x\|)$,

$$
\begin{aligned}
\left|{ }^{c} D^{\beta} h(t)\right| \leq & \frac{1}{\Gamma(\alpha-\beta)} \int_{0}^{t}(t-s)^{\alpha-\beta-1}|v(s)| d s \\
& +\left|\frac{(1-\nu) t^{1-\beta}}{\gamma \Gamma(\alpha) \Gamma(2-\beta)}\right| \int_{0}^{\eta} \int_{0}^{s}(s-m)^{\alpha-1}|v(m)| d m d s \\
& +\left|\frac{(1-\eta) t^{1-\beta}}{\gamma \Gamma(\alpha) \Gamma(2-\beta)}\right| \int_{0}^{1}(1-s)^{\alpha-1}|v(s)| d s \\
& +\left|\frac{(\eta-1) t^{1-\beta}}{\gamma \Gamma(\alpha) \Gamma(2-\beta)}\right| \int_{0}^{\nu} \int_{0}^{s}(s-m)^{\alpha-1}|v(m)| d m d s \\
& +\left|\frac{(1-\eta) t^{1-\beta}}{\gamma \Gamma(\alpha-\beta) \Gamma(2-\beta)}\right| \int_{0}^{1}(1-s)^{\alpha-\beta-1}|v(s)| d s \\
& +\left|\frac{(1-\eta) t^{1-\beta}}{\gamma \Gamma(\alpha-1) \Gamma(2-\beta)}\right| \int_{0}^{1}(1-s)^{\alpha-2}|v(s)| d s \\
\leq & \Lambda_{2}\|p\|_{\infty} \psi(\|x\|)
\end{aligned}
$$

and

$$
\begin{aligned}
\left|h^{\prime}(t)\right| \leq & \frac{1}{\Gamma(\alpha-1)} \int_{0}^{t}(t-s)^{\alpha-2}|v(s)| d s+\left|\frac{(1-\nu)}{\gamma \Gamma(\alpha)}\right| \int_{0}^{\eta} \int_{0}^{s}(s-m)^{\alpha-1}|v(m)| d m d s \\
& +\left|\frac{(1-\eta)}{\gamma \Gamma(\alpha)}\right| \int_{0}^{1}(1-s)^{\alpha-1}|v(s)| d s+\left|\frac{(\eta-1)}{\gamma \Gamma(\alpha)}\right| \int_{0}^{\nu} \int_{0}^{s}(s-m)^{\alpha-1}|v(m)| d m d s \\
& +\left|\frac{(1-\eta)}{\gamma \Gamma(\alpha-\beta)}\right| \int_{0}^{1}(1-s)^{\alpha-\beta-1}|v(s)| d s+\left|\frac{(1-\eta)}{\gamma \Gamma(\alpha-1)}\right| \int_{0}^{1}(1-s)^{\alpha-2}|v(s)| d s
\end{aligned}
$$




$$
\leq \Lambda_{3}\|p\|_{\infty} \psi(\|x\|)
$$

for all $t \in J$, where $\|p\|_{\infty}=\sup _{t \in J}|p(t)|$,

$$
\begin{aligned}
\Lambda_{1}= & {\left[\frac{1}{\Gamma(\alpha+1)}+\frac{\eta^{\alpha+1}}{\Gamma(\alpha+2)(1-\eta)}+\left|\frac{\left(\eta^{2}-2\right)(\nu-1) \eta^{\alpha+1}}{2 \gamma \Gamma(\alpha+2)}\right|+\left|\frac{\left(\eta^{2}-2\right)(\eta-1)}{2 \gamma \Gamma(\alpha+1)}\right|\right.} \\
& +\left|\frac{\left(\eta^{2}-2\right)(1-\eta) \nu^{\alpha+1}}{2 \gamma \Gamma(\alpha+2)}\right|+\left|\frac{\left(\eta^{2}-2\right)(\eta-1)}{2 \gamma \Gamma(\alpha-\beta+1)}\right|+\left|\frac{\left(\eta^{2}-2\right)(\eta-1)}{2 \gamma \Gamma(\alpha)}\right| \\
& +\left|\frac{(1-\nu) \eta^{\alpha+1}}{\gamma \Gamma(\alpha+2)}\right|+\left|\frac{(1-\eta)}{\gamma \Gamma(\alpha+1)}\right|+\left|\frac{(\eta-1) \nu^{\alpha+1}}{\gamma \Gamma(\alpha+2)}\right|+\left|\frac{(1-\eta)}{\gamma \Gamma(\alpha-\beta+1)}\right| \\
& \left.+\left|\frac{(1-\eta)}{\gamma \Gamma(\alpha)}\right|\right], \\
\Lambda_{2}= & {\left[\frac{1}{\Gamma(\alpha-\beta+1)}+\left|\frac{(1-\nu) \eta^{\alpha+1}}{\gamma \Gamma(\alpha+2) \Gamma(2-\beta)}\right|+\left|\frac{(1-\eta)}{\gamma \Gamma(\alpha+1) \Gamma(2-\beta)}\right|\right.} \\
& \left.+\left|\frac{(\eta-1) \nu^{\alpha+1}}{\gamma \Gamma(\alpha+2) \Gamma(2-\beta)}\right|+\left|\frac{(1-\eta)}{\gamma \Gamma(\alpha-\beta+1) \Gamma(2-\beta)}\right|+\left|\frac{(1-\eta)}{\gamma \Gamma(\alpha) \Gamma(2-\beta)}\right|\right], \\
\Lambda_{3}= & {\left[\frac{1}{\Gamma(\alpha)}+\left|\frac{(1-\nu) \eta^{\alpha+1}}{\gamma \Gamma(\alpha+2)}\right|+\left|\frac{(1-\eta)}{\gamma \Gamma(\alpha+1)}\right|+\left|\frac{(\eta-1) \nu^{\alpha+1}}{\gamma \Gamma(\alpha+2)}\right|+\left|\frac{(1-\eta)}{\gamma \Gamma(\alpha-\beta+1)}\right|\right.} \\
& \left.+\left|\frac{(1-\eta)}{\gamma \Gamma(\alpha)}\right|\right] .
\end{aligned}
$$

Hence,

$$
\|h\|=\max _{t \in J}|h(t)|+\left.\max _{t \in J}\right|^{c} D^{\beta} h(t)\left|+\max _{t \in J}\right| h^{\prime}(t) \mid \leq\left(\Lambda_{1}+\Lambda_{2}+\Lambda_{3}\right)\|p\|_{\infty} \psi(\|x\|) .
$$

Now, we show that $N$ maps bounded sets into equi-continuous subsets of $X$. Let $x \in B_{r}$ and $t_{1}, t_{2} \in J$ with $t_{1}<t_{2}$. Then, we have

$$
\begin{aligned}
\left|h\left(t_{2}\right)-h\left(t_{1}\right)\right|= & \mid \frac{1}{\Gamma(\alpha)} \int_{0}^{t_{2}}\left(t_{2}-s\right)^{\alpha-1} v(s) d s-\frac{1}{\Gamma(\alpha)} \int_{0}^{t_{1}}\left(t_{1}-s\right)^{\alpha-1} v(s) d s \\
& +\frac{(1-\nu) t_{2}}{\gamma \Gamma(\alpha)} \int_{0}^{\eta} \int_{0}^{s}(s-m)^{\alpha-1} v(m) d m d s \\
& -\frac{(1-\nu) t_{1}}{\gamma \Gamma(\alpha)} \int_{0}^{\eta} \int_{0}^{s}(s-m)^{\alpha-1} v(m) d m d s \\
& +\frac{(1-\eta) t_{2}}{\gamma \Gamma(\alpha)} \int_{0}^{1}(1-s)^{\alpha-1} v(s) d s-\frac{(1-\eta) t_{1}}{\gamma \Gamma(\alpha)} \int_{0}^{1}(1-s)^{\alpha-1} v(s) d s \\
& \times \frac{(\eta-1) t_{2}}{\gamma \Gamma(\alpha)} \int_{0}^{\nu} \int_{0}^{s}(s-m)^{\alpha-1} v(m) d m d s \\
& -\frac{(\eta-1) t_{1}}{\gamma \Gamma(\alpha)} \int_{0}^{\nu} \int_{0}^{s}(s-m)^{\alpha-1} v(m) d m d s
\end{aligned}
$$




$$
\begin{gathered}
+\frac{(1-\eta) t_{2}}{\gamma \Gamma(\alpha-\beta)} \int_{0}^{1}(1-s)^{\alpha-\beta-1} v(s) d s \\
-\frac{(1-\eta) t_{1}}{\gamma \Gamma(\alpha-\beta)} \int_{0}^{1}(1-s)^{\alpha-\beta-1} v(s) d s \\
+\frac{(1-\eta) t_{2}}{\gamma \Gamma(\alpha-1)} \int_{0}^{1}(1-s)^{\alpha-2} v(s) d s-\frac{(1-\eta) t_{1}}{\gamma \Gamma(\alpha-1)} \int_{0}^{1}(1-s)^{\alpha-2} v(s) d s \mid \\
\leq\|p\|_{\infty} \psi(\|x\|)|| \frac{t_{2}^{\alpha}-t_{1}^{\alpha}}{\Gamma(\alpha+1)}|+| \frac{(1-\nu) \eta^{\alpha+1}\left(t_{2}-t_{1}\right)}{\gamma \Gamma(\alpha+2)}|+| \frac{(1-\eta)\left(t_{2}-t_{1}\right)}{\gamma \Gamma(\alpha+1)} \mid \\
\left.+\left|\frac{(\eta-1) \nu^{\alpha+1}\left(t_{2}-t_{1}\right)}{\gamma \Gamma(\alpha+2)}\right|+\left|\frac{(1-\eta)\left(t_{2}-t_{1}\right)}{\gamma \Gamma(\alpha-\beta+1)}\right|+\left|\frac{(1-\eta)\left(t_{2}-t_{1}\right)}{\gamma \Gamma(\alpha)}\right|\right], \\
\quad{ }^{c} D^{\beta} h\left(t_{2}\right)-{ }^{c} D^{\beta} h\left(t_{1}\right)\left|\leq\|p\|_{\infty} \psi(\|x\|)\right|\left|\frac{t_{2}^{\alpha-\beta}-t_{1}^{\alpha-\beta} \mid}{\Gamma(\alpha-\beta+1)}\right|+\left|\frac{\left(t_{2}^{1-\beta}-t_{1}^{1-\beta}\right)(1-\nu) \eta^{\alpha+1}}{\gamma \Gamma(\alpha+2) \Gamma(2-\beta)}\right| \\
\quad\left|\frac{\left(t_{2}^{1-\beta}-t_{1}^{1-\beta}\right)(1-\eta)}{\gamma \Gamma(\alpha+1) \Gamma(2-\beta)}\right|+\left|\frac{\left(t_{2}^{1-\beta}-t_{1}^{1-\beta}\right)(\eta-1) \nu^{\alpha+1}}{\gamma \Gamma(\alpha+2) \Gamma(2-\beta)}\right| \\
\left.\quad\left|\frac{\left(t_{2}^{1-\beta}-t_{1}^{1-\beta}\right)(1-\eta)}{\gamma \Gamma(\alpha-\beta+1) \Gamma(2-\beta)}\right|+\left|\frac{\left(t_{2}^{1-\beta}-t_{1}^{1-\beta}\right)(1-\eta)}{\gamma \Gamma(\alpha) \Gamma(2-\beta)}\right|\right]
\end{gathered}
$$

and $\left|h^{\prime}\left(t_{2}\right)-h^{\prime}\left(t_{1}\right)\right| \leq\|p\|_{\infty} \psi(\|x\|) \frac{\left(t_{2}^{\alpha-1}-t_{1}^{\alpha-1}\right)}{\Gamma(\alpha)}$ for all $h \in N(x)$. Hence, $\lim _{t_{2} \rightarrow t_{1}} \mid h\left(t_{2}\right)-$ $h\left(t_{1}\right)\left|=\lim _{t_{2} \rightarrow t_{1}}\right|{ }^{c} D^{\beta} h\left(t_{2}\right)-{ }^{c} D^{\beta} h\left(t_{1}\right)\left|=\lim _{t_{2} \rightarrow t_{1}}\right| h^{\prime}\left(t_{2}\right)-h^{\prime}\left(t_{1}\right) \mid=0$ and so by using the Arzela-Ascoli theorem, $N$ is completely continuous. Now, we show that $N$ has a closed graph. Let $x_{n} \rightarrow x_{0}, h_{n} \in N\left(x_{n}\right)$ for all $n$ and $h_{n} \rightarrow h_{0}$. We prove that $h_{0} \in N\left(x_{0}\right)$. For each $n$, choose $v_{n} \in S_{F, x_{n}}$ such that $h_{n}(t)=I^{\alpha} v_{n}(t)-c_{0 v_{n}}-c_{1 v_{n}} t$ for all $t \in J$. Consider the continuous linear operator $\theta: L^{1}(J, \mathbb{R}) \rightarrow X$ defined by $\theta(v)(t)=I^{\alpha} v(t)-c_{0 v}-c_{1 v} t$. By using Lemma 1.1, $\theta o S_{F}$ is a closed graph operator. Since $x_{n} \rightarrow x_{0}$ and $h_{n} \in \theta\left(S_{F, x_{n}}\right)$ for all $n$, there exists $v_{0} \in S_{F, x_{0}}$ such that $h_{0}(t)=$ $I^{\alpha} v_{0}(t)-c_{0 v}-c_{1 v_{0}} t$. Thus, $N$ has a closed graph. In this section, we show that $N(x)$ is convex for all $x \in \mathcal{X}$. Let $h_{1}, h_{2} \in N(x)$ and $w \in[0,1]$. Choose $v_{1}, v_{2} \in S_{F, x}$ such that $h_{i}(t)=I^{\alpha} v_{i}(t)-c_{0 v_{i}}-c_{1 v_{i}} t$ for almost all $t \in J$ and $i=1,2$. Then,

$$
\begin{aligned}
& \left.w h_{1}+(1-w) h_{2}\right](t) \\
= & \frac{1}{\Gamma(\alpha)} \int_{0}^{t}(t-s)^{\alpha-1}\left[w v_{1}(s)+(1-w) v_{2}(s)\right] d s \\
& +\frac{1}{\Gamma(\alpha)(1-\eta)} \int_{0}^{\eta} \int_{0}^{s}(s-m)^{\alpha-1}\left[w v_{1}(m)+(1-w) v_{2}(m)\right] d m d s \\
& +\frac{\left(\eta^{2}-2\right)(\nu-1)}{2 \gamma \Gamma(\alpha)} \int_{0}^{\eta} \int_{0}^{s}(s-m)^{\alpha-1}\left[w v_{1}(m)+(1-w) v_{2}(m)\right] d m d s
\end{aligned}
$$




$$
\begin{aligned}
& +\frac{\left(\eta^{2}-2\right)(\eta-1)}{2 \gamma \Gamma(\alpha)} \int_{0}^{1}(1-s)^{\alpha-1}\left[w v_{1}(s)+(1-w) v_{2}(s)\right] d s \\
& +\frac{\left(\eta^{2}-2\right)(1-\eta)}{2 \gamma \Gamma(\alpha)} \int_{0}^{\nu} \int_{0}^{s}(s-m)^{\alpha-1}\left[w v_{1}(m)+(1-w) v_{2}(m)\right] d m d s \\
& +\frac{\left(\eta^{2}-2\right)(\eta-1)}{2 \gamma \Gamma(\alpha-\beta)} \int_{0}^{1}(1-s)^{\alpha-\beta-1}\left[w v_{1}(s)+(1-w) v_{2}(s)\right] d s \\
& +\frac{\left(\eta^{2}-2\right)(\eta-1)}{2 \gamma \Gamma(\alpha-1)} \int_{0}^{1}(1-s)^{\alpha-2}\left[w v_{1}(s)+(1-w) v_{2}(s)\right] d s \\
& +\frac{(1-\nu) t}{\gamma \Gamma(\alpha)} \int_{0}^{\eta} \int_{0}^{s}(s-m)^{\alpha-1}\left[w v_{1}(m)+(1-w) v_{2}(m)\right] d m d s \\
& +\frac{(1-\eta) t}{\gamma \Gamma(\alpha)} \int_{0}^{1}(1-s)^{\alpha-1}\left[w v_{1}(s)+(1-w) v_{2}(s)\right] d s \\
& +\frac{(\eta-1) t}{\gamma \Gamma(\alpha)} \int_{0}^{\nu} \int_{0}^{s}(s-m)^{\alpha-1}\left[w v_{1}(m)+(1-w) v_{2}(m)\right] d m d s \\
& +\frac{(1-\eta) t}{\gamma \Gamma(\alpha-\beta)} \int_{0}^{1}(1-s)^{\alpha-\beta-1}\left[w v_{1}(s)+(1-w) v_{2}(s)\right] d s \\
& +\frac{(1-\eta) t}{\gamma \Gamma(\alpha-1)} \int_{0}^{1}(1-s)^{\alpha-2}\left[w v_{1}(s)+(1-w) v_{2}(s)\right] d s,
\end{aligned}
$$

for all $t \in J$. Since $F$ has convex values, $S_{F, x}$ is convex and so $w h_{1}+(1-w) h_{2} \in N(x)$. If there exists $\lambda \in(0,1)$ such that $x \in \lambda N(x)$, then there exists $v \in S_{F, x}$ such that $x(t)=I^{\alpha} v(t)-c_{0 v}-c_{1 v} t$ for almost all $t \in J$. Choose $L>0$ such that $\frac{L}{\left(\Lambda_{1}+\Lambda_{2}+\Lambda_{3}\right)\|p\|_{\infty} \psi(\|x\|)}>1$ for all $x \in X$. Thus, $\|x\|<L$. Now, put $U=\{x \in \mathcal{X}:\|x\|<$ $L+1\}$. Note that, there are no $x \in \partial U$ and $\lambda \in(0,1)$ such that $x \in \lambda N(x)$ and the operator $N: \bar{U} \rightarrow P_{c p, c v}(\bar{U})$ is upper semi-continuous because it is completely continuous. Now by using Lemma $1.2, N$ has a fixed point in $\bar{U}$ which is a solution of the inclusion problem (1.1). This completes the proof.

Here, we provide an example for the result.

Example 2.1. Consider the fractional differential inclusion

$$
{ }^{c} D^{\frac{5}{2}} x(t) \in F\left(t, x(t),{ }^{c} D^{\frac{1}{2}} x(t), x^{\prime}(t)\right),
$$

with the boundary value conditions

$$
x(0)+x^{\prime}(0)+{ }^{c} D^{\frac{1}{2}} x(0)=\int_{0}^{\frac{1}{2}} x(s) d s \text { and } x(1)+x^{\prime}(1)+{ }^{c} D^{\frac{1}{2}} x(1)=\int_{0}^{\frac{1}{3}} x(s) d s .
$$

Put, $\alpha=\frac{5}{2}, \beta=\frac{1}{2}, \eta=\frac{1}{2}, \nu=\frac{1}{3}$ and consider the multifunction $F: J \times \mathbb{R}^{3} \rightarrow 2^{\mathbb{R}}$ defined by

$$
F\left(t, x_{1}, x_{2}, x_{3}\right)=\left[\cos t+\frac{\left|x_{1}\right|}{1+\left|x_{1}\right|}+\sin x_{2}, 5+t^{2}+\frac{t}{1+e^{\left|x_{3}\right|}}\right] .
$$


Note that, $\left\|F\left(t, x_{1}, x_{2}, x_{3}\right)\right\|=\sup \left\{|y|: y \in F\left(t, x_{1}, x_{2}, x_{3}\right)\right\} \leq 7$. If $p(t)=1$, and $\psi(t)=7$, then one can check that the assumptions of Theorem 2.1 hold and so the problem (2.1) has at least one solution.

Here, we provide another result about the existence of solutions for the problem (1.1) by changing the assumption of convex values for the multifunction.

Theorem 2.2. Let $m \in C\left(J, \mathbb{R}^{+}\right)$be such that $\|m\|_{\infty}\left(\Lambda_{1}+\Lambda_{2}+\Lambda_{3}\right)<1$. Suppose that $F: J \times \mathbb{R} \times \mathbb{R} \times \mathbb{R} \rightarrow P_{c p}(\mathbb{R})$ is an integrable bounded multifunction such that the map $t \vdash F(t, x, y, z)$ is measurable and $H_{d}\left(F\left(t, x_{1}, x_{2}, x_{3}\right), F\left(t, y_{1}, y_{2}, y_{3}\right)\right) \leq m(t)\left(\left|x_{1}-y_{1}\right|+\right.$ $\left.\left|x_{2}-y_{2}\right|+\left|x_{3}-y_{3}\right|\right)$ for almost all $t \in J$ and $x, y, z, x_{1}, x_{2}, x_{3}, y_{1}, y_{2}, y_{3} \in \mathbb{R}$. Then the problem $(*)$ has a solution.

Proof. Note that, the multivalued map $t \vdash F\left(t, x(t),{ }^{c} D^{B} x(t), x^{\prime}(t)\right)$ is measurable and closed valued for all $x \in \mathcal{X}$. Hence, it has a measurable selection and so the set $S_{F, x}$ is nonempty. Now, consider the operator $N: X \rightarrow 2^{x}$ defined by

$N(x)=\left\{h \in X:\right.$ there exists $v \in S_{F, x}$ such that $\left.h(t)=I^{\alpha} v(t)-c_{0 v}-c_{1 v} t, t \in J\right\}$,

for all $t \in J$. First, we show that $N(x)$ is a closed subset of $X$ for all $x \in X$. Let $x \in \mathcal{X}$ and $\left\{u_{n}\right\}_{n \geq 1}$ be a sequence in $N(x)$ with $u_{n} \rightarrow u$. For each $n$, choose $v_{n} \in S_{F, x}$ such that $u_{n}(t)=I^{\alpha} v_{n}(t)-c_{0 v_{n}}-c_{1 v_{n}} t$ for almost all $t \in J$. Since $F$ has compact values, $\left\{v_{n}\right\}_{n \geq 1}$ has a subsequence which converges to some $v \in L^{1}(J, \mathbb{R})$. Denote the subsequence again by $\left\{v_{n}\right\}_{n \geq 1}$. It is easy to check that $v \in S_{F, x}$ and $u_{n}(t) \rightarrow u(t)=I^{\alpha} v(t)-c_{0 v}-c_{1 v} t$ for all $t \in J$. This implies that $u \in N(x)$. Thus, the multifunction $N$ has closed values. Now, we show that $N$ is a contractive multifunction with constant $l:=\|m\|_{\infty}\left(\Lambda_{1}+\Lambda_{2}+\Lambda_{3}\right)<1$. Let $x, y \in \mathcal{X}$ and $h_{1} \in N(y)$. Choose $v_{1} \in S_{F, y}$ such that $h_{1}(t)=I^{\alpha} v_{1}(t)-c_{0 v_{1}}-c_{1 v_{1}} t$ for almost all $t \in J$. Since

$$
\begin{aligned}
& H_{d}\left(F\left(t, x(t),{ }^{c} D^{\beta} x(t), x^{\prime}(t)\right), F\left(t, y(t),{ }^{c} D^{\beta} y(t), y^{\prime}(t)\right)\right. \\
\leq & m(t)\left(|x(t)-y(t)|+\left|{ }^{c} D^{\beta} x(t)-{ }^{c} D^{\beta} y(t)\right|+\left|x^{\prime}(t)-y^{\prime}(t)\right|\right),
\end{aligned}
$$

for almost all $t \in J$, there exists $w \in F\left(t, x(t),{ }^{c} D^{\beta} x(t), x^{\prime}(t)\right)$ such that

$$
\left|v_{1}(t)-w\right| \leq m(t)\left(|x(t)-y(t)|+\left|{ }^{c} D^{\beta} x(t)-{ }^{c} D^{\beta} y(t)\right|+\left|x^{\prime}(t)-y^{\prime}(t)\right|\right),
$$

for almost all $t \in J$. Define the multifunction $U: J \rightarrow 2^{\mathbb{R}}$ by

$$
\begin{aligned}
U(t)= & \left\{w \in \mathbb{R}:\left|v_{1}(t)-w\right| \leq m(t)\left(|x(t)-y(t)|+\left|{ }^{c} D^{\beta} x(t)-{ }^{c} D^{\beta} y(t)\right|\right.\right. \\
& \left.\left.+\left|x^{\prime}(t)-y^{\prime}(t)\right|\right) \text { for almost all } t \in J\right\} .
\end{aligned}
$$

It is easy to check that the multifunction $U(.) \cap F\left(., x(),.{ }^{c} D^{\beta} x(),. x^{\prime}().\right)$ is measurable. Thus, we can choose $v_{2} \in S_{F, x}$ such that

$$
\left|v_{1}(t)-v_{2}(t)\right| \leq m(t)\left(|x(t)-y(t)|+\left|{ }^{c} D^{\beta} x(t)-{ }^{c} D^{\beta} y(t)\right|+\left|x^{\prime}(t)-y^{\prime}(t)\right|\right),
$$


for almost all $t \in J$. Now, consider $h_{2} \in N(x)$ which is defined by $h_{2}(t)=I^{\alpha} v(t)-$ $c_{0 v_{2}}-c_{1 v_{2}} t$. Hence, we get

$$
\begin{aligned}
& \left|h_{1}(t)-h_{2}(t)\right| \leq \frac{1}{\Gamma(\alpha)} \int_{0}^{t}(t-s)^{\alpha-1}\left|v_{1}(s)-v_{2}(s)\right| d s \\
& +\frac{1}{\Gamma(\alpha)(1-\eta)} \int_{0}^{\eta} \int_{0}^{s}(s-m)^{\alpha-1}\left|v_{1}(m)-v_{2}(m)\right| d m d s \\
& +\left|\frac{\left(\eta^{2}-2\right)(\nu-1)}{2 \gamma \Gamma(\alpha)}\right| \int_{0}^{\eta} \int_{0}^{s}(s-m)^{\alpha-1}\left|v_{1}(m)-v_{2}(m)\right| d m d s \\
& +\left|\frac{\left(\eta^{2}-2\right)(\eta-1)}{2 \gamma \Gamma(\alpha)}\right| \int_{0}^{1}(1-s)^{\alpha-1}\left|v_{1}(s)-v_{2}(s)\right| d s \\
& +\left|\frac{\left(\eta^{2}-2\right)(1-\eta)}{2 \gamma \Gamma(\alpha)}\right| \int_{0}^{\nu} \int_{0}^{s}(s-m)^{\alpha-1}\left|v_{1}(m)-v_{2}(m)\right| d m d s \\
& +\left|\frac{\left(\eta^{2}-2\right)(\eta-1)}{2 \gamma \Gamma(\alpha-\beta)}\right| \int_{0}^{1}(1-s)^{\alpha-\beta-1}|v(s)| d s \\
& +\left|\frac{\left(\eta^{2}-2\right)(\eta-1)}{2 \gamma \Gamma(\alpha-1)}\right| \int_{0}^{1}(1-s)^{\alpha-2}\left|v_{1}(s)-v_{2}(s)\right| d s \\
& +\left|\frac{(1-\nu) t}{\gamma \Gamma(\alpha)}\right| \int_{0}^{\eta} \int_{0}^{s}(s-m)^{\alpha-1}\left|v_{1}(m)-v_{2}(m)\right| d m d s \\
& +\left|\frac{(1-\eta) t}{\gamma \Gamma(\alpha)}\right| \int_{0}^{1}(1-s)^{\alpha-1}\left|v_{1}(s)-v_{2}(s)\right| d s \\
& +\left|\frac{(\eta-1) t}{\gamma \Gamma(\alpha)}\right| \int_{0}^{\nu} \int_{0}^{s}(s-m)^{\alpha-1}\left|v_{1}(m)-v_{2}(m)\right| d m d s \\
& +\left|\frac{(1-\eta) t}{\gamma \Gamma(\alpha-\beta)}\right| \int_{0}^{1}(1-s)^{\alpha-\beta-1}\left|v_{1}(s)-v_{2}(s)\right| d s \\
& +\left|\frac{(1-\eta) t}{\gamma \Gamma(\alpha-1)}\right| \int_{0}^{1}(1-s)^{\alpha-2}\left|v_{1}(s)-v_{2}(s)\right| d s \\
& \leq \Lambda_{1}\|m\|_{\infty}\|x-y\| \text {, } \\
& \left|{ }^{c} D^{\beta} h_{1}(t)-{ }^{c} D^{\beta} h_{2}(t)\right| \leq \frac{1}{\Gamma(\alpha-\beta)} \int_{0}^{t}(t-s)^{\alpha-\beta-1}\left|v_{1}(s)-v_{2}(s)\right| d s \\
& +\left|\frac{(1-\nu) t^{1-\beta}}{\gamma \Gamma(\alpha) \Gamma(2-\beta)}\right| \int_{0}^{\eta} \int_{0}^{s}(s-m)^{\alpha-1}\left|v_{1}(m)-v_{2}(m)\right| d m d s \\
& +\left|\frac{(1-\eta) t^{1-\beta}}{\gamma \Gamma(\alpha) \Gamma(2-\beta)}\right| \int_{0}^{1}(1-s)^{\alpha-1}\left|v_{1}(s)-v_{2}(s)\right| d s \\
& +\left|\frac{(\eta-1) t^{1-\beta}}{\gamma \Gamma(\alpha) \Gamma(2-\beta)}\right| \int_{0}^{\nu} \int_{0}^{s}(s-m)^{\alpha-1}\left|v_{1}(m)-v_{2}(m)\right| d m d s
\end{aligned}
$$




$$
\begin{aligned}
& \quad+\left|\frac{(1-\eta) t^{1-\beta}}{\gamma \Gamma(\alpha-\beta) \Gamma(2-\beta)}\right| \int_{0}^{1}(1-s)^{\alpha-\beta-1}\left|v_{1}(s)-v_{2}(s)\right| d s \\
& +\left|\frac{(1-\eta) t^{1-\beta}}{\gamma \Gamma(\alpha-1) \Gamma(2-\beta)}\right| \int_{0}^{1}(1-s)^{\alpha-2}\left|v_{1}(s)-v_{2}(s)\right| d s \\
& \leq \Lambda_{2}\|m\|_{\infty}\|x-y\|
\end{aligned}
$$

and

$$
\begin{aligned}
\left|h_{1}^{\prime}(t)-h_{2}^{\prime}(t)\right| \leq & \frac{1}{\Gamma(\alpha-1)} \int_{0}^{t}(t-s)^{\alpha-2}\left|v_{1}(s)-v_{2}(s)\right| d s \\
& +\left|\frac{(1-\nu)}{\gamma \Gamma(\alpha)}\right| \int_{0}^{\eta} \int_{0}^{s}(s-m)^{\alpha-1}\left|v_{1}(m)-v_{2}(m)\right| d m d s \\
& +\left|\frac{(1-\eta)}{\gamma \Gamma(\alpha)}\right| \int_{0}^{1}(1-s)^{\alpha-1}\left|v_{1}(s)-v_{2}(s)\right| d s \\
& +\left|\frac{(\eta-1)}{\gamma \Gamma(\alpha)}\right| \int_{0}^{\nu} \int_{0}^{s}(s-m)^{\alpha-1}\left|v_{1}(m)-v_{2}(m)\right| d m d s \\
& +\left|\frac{(1-\eta)}{\gamma \Gamma(\alpha-\beta)}\right| \int_{0}^{1}(1-s)^{\alpha-\beta-1}\left|v_{1}(s)-v_{2}(s)\right| d s \\
& +\left|\frac{(1-\eta)}{\gamma \Gamma(\alpha-1)}\right| \int_{0}^{1}(1-s)^{\alpha-2}\left|v_{1}(s)-v_{2}(s)\right| d s \\
\leq & \Lambda_{3}\|m\|_{\infty}\|x-y\|
\end{aligned}
$$

and so $\left\|h_{1}-h_{2}\right\| \leq\left(\Lambda_{1}+\Lambda_{2}+\Lambda_{3}\right)\|m\|_{\infty}\|x-y\|=l\|x-y\|$. This implies that the multifunction $N$ is a contraction with closed values. Thus by using the result of Covitz and Nadler, $N$ has a fixed point which is a solution for the inclusion problem (1.1).

Next example illustrates last result.

Example 2.2. Consider the inclusion problem

$$
{ }^{c} D^{\frac{7}{3}} x(t) \in F\left(t, x(t),{ }^{c} D^{\frac{1}{3}} x(t), x^{\prime}(t)\right),
$$

with the boundary value conditions

$$
x(0)+x^{\prime}(0)+{ }^{c} D^{\frac{1}{3}} x(0)=\int_{0}^{\frac{1}{2}} x(s) d s \text { and } x(1)+x^{\prime}(1)+{ }^{c} D^{\frac{1}{3}} x(1)=\int_{0}^{\frac{1}{3}} x(s) d s .
$$

Put $\alpha=\frac{7}{3}, \beta=\frac{1}{3}, \eta=\frac{1}{2}, \nu=\frac{1}{3}$ and consider the multifunction $F: J \times \mathbb{R}^{3} \rightarrow 2^{\mathbb{R}}$ defined by

$$
F\left(t, x_{1}, x_{2}, x_{3}\right)=\left[0, \frac{t \sin x_{1}}{15\left(5+3 t^{2}\right)}+\frac{t\left|x_{2}\right|}{100\left(1+\left|x_{2}\right|\right)}+\frac{\left|x_{3}\right|}{100\left(1+\left|x_{3}\right|\right)}\right] .
$$


It is easy to see that

$$
H_{d}\left(F\left(t, x_{1}, x_{2}, x_{3}\right), F\left(t, y_{1}, y_{2}, y_{3}\right)\right) \leq\left(\frac{t}{15\left(5+30 t^{2}\right)}+\frac{t}{100}+\frac{1}{100}\right) \sum_{i=1}^{3}\left|x_{i}-y_{i}\right|,
$$

for all $t \in J$ and $x_{1}, x_{2}, x_{3}, y_{1}, y_{2}, y_{3} \in \mathbb{R}$. If $m(t)=\frac{t}{15\left(5+3 t^{2}\right)}+\frac{t}{100}+\frac{1}{100}$ for all $t \in J$, then $H\left(F\left(t, x_{1}, x_{2}, x_{3}\right), F\left(t, y_{1}, y_{2}, y_{3}\right)\right) \leq m(t) \sum_{i=1}^{3}\left|x_{i}-y_{i}\right|$. On the other hand, we have $L=\|m\|_{\infty}\left(\Lambda_{1}+\Lambda_{2}+\Lambda_{3}\right) \leq 0.0333 \times(3.654+1.263+0.963) \simeq 0.19580<1$. Thus, the assumptions of Theorem 2.2 hold and so the inclusion problem (2.2) has at least one solution.

\section{REFERENCES}

[1] R. P. Agarwal and B. Ahmad, Existence theory for anti-periodic boundary value problems of fractional differential equations and inclusions, J. Appl. Math. Comput. 62 (2011), 1200-1214.

[2] R. P. Agarwal, M. Belmekki and M. Benchohra, A survey on semilinear differential equations and inclusions invovling Riemann-Liouville fractional derivative, Adv. Differential Equations (2009), Article ID 981728.

[3] R. P. Agarwal, M. Benchohra and S. Hamani, A survey on existence results for boundary value problems of nonlinear fractional differential equations and inclusions, Acta Appl. Math. 109 (2010), 973-1033.

[4] B. Ahmad and S. K. Ntouyas, Boundary value problem for fractional differential inclusions with four-point integral boundary conditions, Surv. Math. Appl. 6 (2011), 175-193.

[5] B. Ahmad, S. K. Ntouyas and A. Alsedi, On fractional differential inclusions with with antiperiodic type integral boundary conditions, Bound. Value Probl. (2013), Article ID 82.

[6] A. Alsaedi, S. K. Ntouyas and B. Ahmad, Existence of solutions for fractional differential inclusions with separated boundary conditions in Banach spaces, Abstr. Appl. Anal. (2013), Article ID 869837.

[7] J. Aubin and A. Ceuina, Differential Inclusions: Set-Valued Maps and Viability Theory, Springer-Verlag, Berlin, 1984.

[8] D. Baleanu, R. P. Agarwal, H. Mohammadi and Sh. Rezapour, Some existence results for a nonlinear fractional differential equation on partially ordered Banach spaces, Bound. Value Probl. (2013), Article ID 112.

[9] D. Baleanu, H. Mohammadi and Sh. Rezapour, The existence of solutions for a nonlinear mixed problem of singular fractional differential equations, Adv. Differential Equations (2013), Article ID 359.

[10] D. Baleanu, H. Mohammadi and Sh. Rezapour, Positive solutions of a boundary value problem for nonlinear fractional differential equations, Abstr. Appl. Anal. (2012), Article ID 837437.

[11] D. Baleanu, H. Mohammadi and Sh. Rezapour, Some existence results on nonlinear fractional differential equations, Philos. Trans. A 371 (2013), Article ID 20120144.

[12] D. Baleanu, H. Mohammadi and Sh. Rezapour, On a nonlinear fractional differential equation on partially ordered metric spaces, Adv. Differential Equations (2013), Article ID 83.

[13] D. Baleanu, H. Mohammadi and Sh. Rezapour, The existence of solutions for a nonlinear mixed problem of singular fractional differential equations, Adv. Differential Equations (2013), Article ID 359.

[14] D. Baleanu, Z. Nazemi and Sh. Rezapour, The existence of positive solutions for a new coupled system of multi-term singular fractional integro-differential boundary value problems, Abstr. Appl. Anal. (2013), Article ID 368659. 
[15] D. Baleanu, Z. Nazemi and Sh. Rezapour, Existence and uniqueness of solutions for multi-term nonlinear fractional integro-differential equations, Adv. Differential Equations (2013), Article ID 368 .

[16] D. Baleanu, Z. Nazemi and Sh. Rezapour, Attractivity for a $k$-dimensional system of fractional functional differential equations and global attractivity for a $k$-dimensional system of nonlinear fractional differential equations, J. Ineq. Appl. (2014), Article ID 31.

[17] V. Berinde and M. Pacurar, The role of the Pompeiu-Hausdorff metric in fixed point theory, Creat. Math. Inform. 22(2) (2013), 35-42.

[18] M. Bragdi, A. Debbouche and D. Baleanu, Existence of solutions for fractional differential inclusions with separated boundary conditions in Banach space, Adv. Math. Phys. (2013), Article ID 426061.

[19] M. Benchohra and N. Hamidi, Fractional order differential inclusions on the Half-Lin, Surv. Math. Appl. 5 (2010), 99-111.

[20] M. Benchohra and S. K. Ntouyas, On second order differential inclusions with periodic boundary conditions, Acta Math. Univ. Comenian. LXIX(2) (2000), 173-181.

[21] H. Covitz, S. Nadler, Multivalued contraction mappings in generalized metric spaces, Israel J. Math. 8 (1970), 5-11.

[22] K. Deimling, Multi-Valued Differential Equations, Walter de Gruyter, Berlin, 1992.

[23] A. M. A. El-Sayed and A. G. Ibrahim, Multivalued fractional differential equations, Appl. Math. Comput. 68 (1995), 15-25.

[24] A. Granas and J. Dugundji, Fixed Point Theory, Springer-Verlag, New York, 2005.

[25] A. A. Kilbas, H. M. Srivastava and J. J. Trujillo, Theory and Applications of Fractional Differential Equations North-Holland Mathematics Studies, Elsevier Science, 2006.

[26] M. Kisielewicz, Differential Inclusions and Optimal Control, Kluwer, Dordrecht, 1991.

[27] K. Q. Lan and W. Lin, Positive solutions of systems of caputo fractioal differential equations, Commun. Appl. Anal. 17(1) (2013), 61-86.

[28] A. Lasota and Z. Opial, An application of the Kakutani-Ky Fan theorem in the theory of ordinary differential equations, Bull. Acad. Pol. Sci. Set. Sci. Math. Astronom. Phy. 13 (1965), 781-786.

[29] X. Liu and Z. Liu, Existence result for fractional differential inclusions with multivalued term depending on lower-order derivative, Abstr. Appl. Anal. (2012), Article ID 423796.

[30] K. S. Miller and B. Ross, An Introduction to the Fractional Calculus and Differential Equations, John Wiley, 1993.

[31] J. J. Nieto, A. Ouahab and P. Prakash, Extremal solutions and relaxation problems for fractional differential inclusions, Abstr. Appl. Anal. (2013), Article ID 292643.

[32] P. D. Phung and L. X. Truong, On a fractional differential inclusion with integral boundary conditions in Banach space, Fract. Calc. Appl. Anal. 16(3) (2013), 538-558.

[33] A. Ouahab, Some results for fractional boundary value problem of differential inclusions, Nonlinear Anal. 69 (2008), 3877-3896.

[34] I. Podlubny, Fractional Differential Equations, Academic Press, 1999.

[35] X. Su, Boundary value problem for a coupled system of nonlinear fractional differential equations, Appl. Math. Lett. 22 (2009), 64-69.

[36] J. Wang and A. G. Ibrahim, Existence and controllability results for nonlocal fractional impulsive differential inclusions in Banach spaces, J. Funct. Spac. Appl. (2013), Article ID 518306.

[37] S. G. Samko, A. A. Kilbas and O. I. Marichev, Fractional Integrals and Derivatives: Theory and Applications, Gordan and Breach, Amsterdam, 1993. 
${ }^{1}$ Department of Medical Research China Medical University Hospital China Medical University

No. 91, HSUEH-ShiH ROAD

TAICHUNG, TAIWAN

E-mail address: sh.rezapour@mail.cmuh.org.tw, sh.rezapour@azaruniv.edu

${ }^{2}$ Department of Mathematics

Azarbaijan Shahid Madani University

TABRIZ, IRAN

E-mail address: v.hedayati1367@gmail.com

*Corresponding Author 